\title{
Les cours de linguistique générale de Ferdinand de Saussure, un projet de Paul-F. Regard et Antoine Meillet
}

\author{
Claudia Mejía Quijano \\ Universidad de Antioquia, Colombia \\ lucia.mejia@udea.edu.co
}

\section{Résumé}

Le livre intitulé Cours de linguistique générale, dont on vient de célébrer le premier siècle de publication, a eu une genèse complexe, qui a convoqué l'effort de nombreuses personnes. Son exégèse est longue et fort variée. Dans cet article, j'aimerais mentionner un projet parallèle au CLG, qui, à l'époque, n'a pas été mené à terme par ses concepteurs et est resté méconnu, mais qui, par la suite, a été spontanément développé par plusieurs linguistes au long du siècle écoulé. J'aborderai notamment le moment de la conception de ce projet de deux excellents disciples de Saussure, grâce à des lettres inédites, récemment retrouvées au Château de Vufflens (Mejía Quijano, 2015). Ces lettres viennent compléter d'autres lettres, déjà publiées quoique de façon éparse, et cet ensemble permet de saisir plus clairement les enjeux théoriques de ce projet parallèle, qui est étonnamment toujours d'actualité.

Mots clés: Antoine Meillet, Paul-F. Regard, lingüística general, Saussure, manuscritos, cursos.

\section{The courses of general linguistics of Ferdinand de Saussure, a project of Paul-F. Regard and Antoine Meillet}

\begin{abstract}
The Course of General Linguistics, of which we have just celebrated the first century of publication, had a complex genesis, which summoned the effort of many people. His exegesis is long and varied. In this article, I would like to mention a project parallel to the CLG, which at the time was not carried out by its designers and remained unknown, but which was subsequently spontaneously developed by several Linguists throughout the last century. I will mention in particular the moment of conception of this project of two excellent disciples of Saussure, thanks to unpublished letters, recently found at the Castle of Vufflens (Mejía Quijano, 2015). These letters are complementary to other letters, which have already been published, albeit in a sparse way, and this set makes it possible to better understand the theoretical stakes of this parallel project, which is surprisingly still current.
\end{abstract}

Keywords: Antoine Meillet, Paul-F. Regard, lingüística general, Saussure, manuscritos, cursos. 
Le livre intitulé Cours de linguistique générale (CLG), dont on vient de célébrer le premier siècle de publication, a eu une genèse complexe, convoquant l'effort de nombreuses personnes; son exégèse a donc été longue et fort variée. Dans cet article, j'aimerais cheminer un sentir non battu en dévoilant un projet parallèle au CLG qui, à l'époque, tout comme quelques autres, est resté méconnu, n'ayant pas été mené à terme par ses concepteurs. Cependant, ce projet concurrent, par la suite, a été spontanément développé par plusieurs linguistes au long du siècle écoulé, ce qui lui confère un intérêt singulier.

J'aborderai notamment le moment de la conception de ce projet grâce à des lettres inédites, récemment retrouvées au Château de Vufflens, lieu de mort de Ferdinand de Saussure (Mejía Quijano, 2015). Ces lettres viennent compléter d'autres lettres, déjà publiées de façon éparse, et l'ensemble de ce dossier épistolaire permet de saisir plus clairement les enjeux théoriques qui ont animé deux disciples de Saussure dans la conception de ce projet qui est, étonnamment, toujours d'actualité.

\section{La perte}

A la mort de Ferdinand de Saussure, tous ses élèves partagent un même sentiment: le regret du savoir, maintenant, perdu avec la disparition du maître, et le besoin de les ressentir encore vivants, tous les deux. En témoigne les nombreuses missives reçues par la veuve de Saussure, comme cette carte de Louis Havet:

24 février 1913

$$
\text { 18, Quai d'Orléans ( } 4^{\mathrm{e}} \text { Arrt.) }
$$

\section{Chère Madame,}

C'est avec une profonde affliction que nous venons d'apprendre, d'abord par notre ami Meillet, puis par une note du Temps, le cruel deuil qui vous frappe. J'avais admiré Ferdinand de Saussure avant de le connaître, admiré avec un vrai enthousiasme, alors quej'avais trente ans et lui vingt-et-un. J'ai découvert ensuite que le linguiste de génie était aussi une belle nature morale, capable d'affection durable et d'amitié fidèle. En remuant mes souvenirs lointains, je retrouve celui de sa visite lors du deuil le plus affreux par lequel j'ai passé (je venais de perdre ma mère). Il y a quatre ans, il venait à Paris exprès pour s'associer à une fête que des amis me donnaient, il y était leur porte-parole avec Meillet, et il me parlait avec tout son cœur. Moi aussi je lui étais fidèle, et, si rares que fussent nos relations par le fait des circonstances extérieures, je pensais souvent à l'homme et au savant, un savant dont le cerveau mûrissait là bas vingt découvertes aujourd'hui perdues sans retour. En vous exprimant ma respectueuse sympathie, Madame, je me soulage moi-même; je n'ose envisager votre douleur et je fais des vœux pour que vos enfants, le temps et le courage vous fassent du bien.

\section{Havet}

Carte inédite, manuscrit appartenant à Jacques et Philippe de Saussure et déposé au Château de Vufflens (Mejía Quijano, 2015).

A ce témoignage écrit, on peut ajouter les discours donnés un peu partout spontanément, dont voici ceux d'Antoine Meillet, au Collège de France et à l'Ecole Pratique des Hautes Études, recueillis par Paul-F. Regard à l'intention de Marie de Saussure:

Le vingt-cinq février mil-neuf-cent-treize, au début de sa leçon au Collège de France, Monsieur le professeur Antoine Meillet a dit en substance:

Avant de commencer, je dois vous annoncer la mort du maître de la grammaire comparée, Monsieur Ferdinand de Saussure, qui n'a pas enseigné ici parce qu'il était étranger mais qui aurait pu entrer dans cette maison s'il l'avait désiré.

Ceux qui connaissent un peu les choses savent quaucun homme, avec Monsieur Bréal, n'a exercé une pareille influence sur le développement de la grammaire comparée en France. On peut dire que tous les linguistes qui se sont formés à ce moment portent sa marque, et non seulement cela, car jamais il n'y a eu une pareille floraison de vocations linguistiques et de linguistes ayant pris chacun, par la suite, sa place originale: Jamais ils ne se sont contentés d'une observation particulière, de la simple observation d'un fait; toujours -c'est ce qui caractérise les élèves de de Saussure- comme lui, ils s'efforcent de poser des systèmes, 
et c'est là la définition-même de la science. Aucun n'a approché le maître sous ce rapport. Son mémoire sur le système primitif des voyelles indo-européennes et ses travaux sur l'accentuation lituanienne sont des modèles! Plus encore, peut-être, que par son œuvre, il a été utile à ses élèves par son influence personnelle. Le nom de Ferdinand de Saussure vivra et restera comme un modèle.

\section{$\overline{-1}$ \\ Relevé pour Madame F. de Saussure \\ Paris, mars 1913 \\ Paul-F. Regard.}

Le vingt-cinq février mil-neuf-cent-treize, à neuf heures du matin, à l'Ecole pratique des Hautes Etudes de la Sorbonne, Monsieur Antoine Meillet a commencé sa leçon à peu près en ces termes:

J'ai à vous annoncer la nouvelle douloureuse de la mort de Monsieur Ferdinand de Saussure qui a occupé cette chaire pendant dix ans, de 1881 à 1891. Personne ne peut savoir ce qu'il a été pour nous. C'est certainement l'intelligence la plus claire qui ait jamais fait pénétrer la lumière en grammaire comparée.

D'autres ont eu une érudition égale. Mais personne n'a jamais su faire le plan des choses comme lui, ni montrer d'une façon exacte comment les faits s'enchaînent les uns aux autres. Son livre -l'ouvrage d'un homme de vingt ans- a été celui qui a mis au point d'une façon définitive tout le vocalisme indoeuropéen, et, comme le vocalisme domine toute la grammaire indo-européenne, cela posait pour toujours le système des langues indo-européennes. Il a paru en 1878 et, aujourd'hui même, quelques détails, très peu seulement, ont vieilli. Pour étudier ce sujet, il n'y a pas eu d'autre moyen, récemment, que de revenir aux idées de Monsieur Ferdinand de Saussure, qui avaient été un peu négligées depuis quelques années, non pas ici mais en Allemagne. Et Monsieur Hirt a pris comme point de départ, pour écrire son livre, l'ouvrage de Monsieur Ferdinand de Saussure.

Mais son organisme était visiblement miné depuis longtemps. Et sa production diminuait d'année en année. Mais jamais sa force intellectuelle n'a fait défaut. Ses travaux sur l'accentuation lituanienne, qui sont venus éclairer aussi l'accent dans les langues slaves, l'ont bien montré! Il est mort prématurément et $l^{\prime}$ on peut dire progressivement. C'est une grande douleur qu'il ait craint souvent d'émettre ses idées. Il avait un sens si exact de ces choses: Nous avons perdu tout ce que Ferdinand de Saussure a pensé et ne nous a pas dit!

\section{Relevé pour Madame F. de Saussure \\ Paris, mars 1913 \\ Paul-F. Regard}

Transcriptions inédites de Paul-F. Regard, envoyées de Paris à Marie de Saussure, le 10 mars 1913 (selon cachet de la Poste). Manuscrits appartenant à Jacques et Philippe de Saussure et déposés au Château de Vufflens

(Mejía Quijano, 2015) ${ }^{1}$.

Paul-F. Regard, linguiste peu connu, avait commencé une thèse sous la direction de Saussure après avoir suivi, entre autres cours du maitre genevois, le deuxième cours de linguistique générale, dont il avait conservé ses cahiers, mis au propre. Au moment du décès de Saussure, Regard se trouve à Paris, où il suit l'enseignement d'Antoine Meillet, lequel est au courant de l'existence des cahiers sur la linguistique générale de Regard. Il semble que c'est dans le dialogue entre disciple et maître que surgit l'idée de publier les cahiers de linguistique générale correspondant au deuxième cours.

\section{Le projet Regard-Meillet}

Pour mieux comprendre leur projet, faisons d'abord connaissance de cet élève particulier. Paul-F. Regard (vers 1888-1962) est fils d'Etienne Regard et Anna-Thérèse, née Cuendet ${ }^{2}$. D'une

1 Les lettres de Paul-F. Regard ici publiées ont été transcrites par Jean-Claude Müller, que je remercie très sincèrement.

2 Je remercie Barbara Roch-Lochner pour son aide dans la recherche biographique et épistolaire concernant ce linguiste. 
famille genevoise modeste et très croyante, c'est un homme sensible et respectueux des traditions, mais en même temps un grand travailleur, féru de science et dont l'intelligence rejoint la systématicité saussurienne, ce qu'on peut constater rétrospectivement par ses travaux publiés, notamment ses thèses (1918 et 1919). Après avoir fini sa licence ès lettres à Genève en $1911^{3}$, il entame une thèse en grec ancien sur les Ecritures, mais il tient à faire un travail de linguiste en se démarquant des théologiens, et trouve chez Saussure le modèle et l'appui qu'il recherche, comme il le dira à Marie de Saussure à la mort de son maître:

Paris, mars 1913

Très honorée Madame,

Le sujet de ma modeste lettre me permet sûrement de vous l'adresser sans m'excuser beaucoup de la grande liberté prise en l'écrivant. Presque tardives, ces lignes paraîtront, peutêtre, l'expression d'une sympathie trop lente au deuil immense qui, dépassant le cadre de l'illustre famille, atteint douloureusement, dans leurs meilleures affections, tant d'amis si respectueux et si reconnaissants partout. C'est que n'ayant pas l'honneur d'être connu de vous personnellement, très honorée Madame, par un sentiment que je sais que vous comprendrez bien, j'ai trouvé mieux d'attendre un peu de temps, avant de prendre la plume. Et c'est dans le silence, jusqu'ici, qu'avec respect et vénération, j'ai mêlé mes propres larmes à vos pleurs...

À partir de mil-neuf-cent-huit, j'ai eu le privilège d'être l'élève attentif de Monsieur le professeur Ferdinand de Saussure. Il m'a, dès le commencement, aidé des encouragements affectueux et des savants conseils qu'il donnait avec une bonté inlassable et une bienveillance toujours croissante. À tant de bienfaits, dont je comprenais tout le prix, j'étais d'autant plus sensible que je m'en sentais moins digne. Dans l'illustre maître que je viens de perdre, l'homme et le professeur étaient inséparables. Aux richesses infinies de sa vaste et profonde science, il joignait le trésor encore plus inestimable de sa noblesse parfaite et de sa rare distinction.

Sa personne offrait à la fois le spectacle le plus admirable et l'exemple le plus éloquent à toute intelligence avide de savoir et à toute âme éprise de beauté. Et, si grande que fût sa maîtrise, unique, dans le domaine scientifique, le gentilhomme, en lui, était plus génial encore que le professeur. Pour toutes ces raisons, je l'aimais profondément. Ses leçons étaient (et sont restées) les seules qui m'inspirassent une confiance sans limites et une admiration sans bornes. Tout y était beau et grand, tout y donnait la science, tout y éveillait l'enthousiasme! D'un vol plané à l'ampleur majestueuse, tous les petits détails de la plaine étaient discernés en considération de l'ensemble des choses par l'œil le plus avisé qui fût au monde. Et, de cette activité créatrice, jaillissaient les lumineuses synthèses et les systématisations géniales. Le grand cours de Linguistique générale, celui de grammaire comparée du grec et du latin, la conférence de linguistique germanique -pour ne parler que de ce dont j'ai l'expérience personnelle-avaient tous pour résultat évident (ces choses-là se sentent d'emblée même par des débutants) de faire parvenir la science et l'enseignement sous leur plus belle expression à leur plus haut idéal. Et personne ne peut savoir ce que j'ai souffert d'être empêché, par les programmes et les questions d'examen qui restreignent la liberté d'une façon affreuse, de suivre, selon mes désirs, toutes ses magistrales leçons et de donner, en particulier, au sanscrit la place qu'il aurait dû avoir dans mes études et que, pressé de toutes parts, j'étais impuissant à lui fournir.

Sans les grades à prendre, le professeur aurait vu plus d'étudiants zêlés profiter de cet enseignement auquel il tenait. Toujours, j'ai beaucoup joui de mes relations avec Monsieur Ferdinand de Saussure, qui m'a, sans cesse, fait l'accueil le plus aimable et qui m’a donné souvent, tant par sa parole admirable que par sa plume habile, les avis les plus remarquablement bienveillants et les plus éminemment utiles. J'ai conservé, avec le plus grand soin, toutes ses lettres ravissantes,

3 Aux Archives de l'Université de Genève, on trouve les notes des étudiants: Paul F. Regard a obtenu 5.5 sur 6.- à l'examen correspondant au cours de Linguistique générale, la meilleure note accordée par Saussure pour cet examen, que le maître genevois a fait passer 5 années durant. 
qui, toutes, portent le reflet magnifique de sa haute distinction d'homme et de savant. Et je serais bien reconnaissant, très-honorée Madame, (si ce n'est pas demander une chose indiscrète ou impossible) qu'une gracieuse faveur de votre part m'accordât la joie d'avoir une photographie de mon illustre maître! Car j'ai aimé et admiré Monsieur le professeur Ferdinand de Saussure, d'une affection très vive et $d^{\prime}$ une admiration sans bornes... Et, toujours, sa personne souverainement noble et distinguée restera vivante dans mon souvenir!

Il me reste encore à vous dire, très-honorée Madame, que j'ai recueilli aussi fidèlement que possible au milieu de l'émotion générale, à votre intention surtout, les paroles par lesquelles Monsieur le Professeur Antoine Meillet a annoncé, à ses auditeurs de l'Ecole pratique des HautesEtudes et du Collège de France, la douloureuse nouvelle de la mort de son maître Monsieur Ferdinand de Saussure. C'est le texte mis au net de ces paroles mémorables que je me permets de vous adresser sous une seconde enveloppe, expédiée par le même courrier que cette lettre.

Veuillez, très honorée Madame, agréer ces faibles lignes dictées par un pieux respect et un réel attachement, et recevoir, par elles, l'hommage particulièrement ému de ma sympathie la plus sincère et la plus profondément respectueuse:

\section{Paul-F. Regard}

Lettre publiée dans les Beiträge zur Gechichte der Sprachwissenschaft 26 (Mejia Quijano, Jaramillo Giraldo et Pérez Zapata «La voix de l'élève dans la parole du maître», 2016). Manuscrit appartenant à Jacques et Philippe de Saussure et déposé au Château de Vufflens (Mejía Quijano, 2015).

La thèse de Regard, Contribution à l'étude des prépositions dans la langue du Nouveau Testament, parue en 1918 à Paris, témoigne de façon étonnante de la marque de Saussure sur cet élève: cet ouvrage suit
admirablementlaméthodestatistiquequeleSaussure indo-européaniste avait utilisée pour ses travaux et représente un modèle du genre, avec en prime la distinction synchronie-diachronie, expliquée par le Saussure généraliste tout particulièrement dans le deuxième cours de linguistique générale, auquel l'auteur a assisté, et appliquée par Regard avec rigueur, comme le signale Meillet dans le compte-rendu qu'il a consacré à cette thèse.

Cependant, en février 1913, Regard n'est qu'au milieu du travail préparatoire de sa thèse et la mort de Saussure le laisse en très mauvaise posture vis-à-vis de son futur académique, comme on peut le constater dans cette lettre qu'il adresse à Charles Bally, quelques semaines après la mort de son directeur de thèse $e^{4}$ :

Paris, le 16 mars 1913

\section{Monsieur et cher Maître,}

Voici, enfin, les deux petites notes que je vous avais promises. Je suis sûr qu'elles vous intéresseront. Meillet m’a dit et répété qu'il a trouvé "admirable" votre article de la Semaine littéraire ${ }^{5}$. Il m’a demandé si je savais ce qui allait se faire à l'Université de Genève pour la succession de Monsieur de Saussure ${ }^{6}$. Je n'ai pu le renseigner que sur les possibilités entrevues, où vous avez, naturellement, au moins la trèsgrosse part, tout en disant que probablement la Faculté des lettres agirait avec lenteur, puisque deux suppléants sont déjà en fonction pour cette année $^{7}$ et qu'elle ne tient vraisemblablement pas à vous obliger le plus possible. Je vous répète, au sujet de votre nomination définitive, mes vœux affectueusement enthousiastes.

A la Société de Linguistique M. Ferrand, président, et, surtout, M. Meillet ont parlé en termes justes et touchants de l'illustre défunt, ce que mentionneront sans doute les Mémoires

4 Bulletin de la Société de Linguistique de Paris 21, 1919: 212-215. Compte-rendu signalé par René Amacker (1989). 5 Hommage à Saussure, paru le 1er mars 1913.

6 Privat-docent à l'Université de Genève, Bally briguait une chaire depuis quelques années avant la mort de Saussure, et sera finalement élu précisément à la chaire de linguistique en juin 1913.

7 Très malade et déjà sans voix, Saussure n'avait pas commencé l'année académique 1912-1913: il a demandé à être remplacé par Albert Sechehaye pour son cours de Linguistique générale et par Charles Bally pour les autres cours (Cf. Lettres de Saussure à F. Decrue (Doyen de la Faculté) et à W. Rosier (Département de l'Instruction Publique), de septembre 1912 (Mejía Quijano, 2014). 
de la Société. Ceux-ci contiendront, d'ailleurs, plus tard, un important article de M. Meillet sur M. Ferdinand de Saussure, article qui paraîtra aussi, sous réserve d'un changement d'avis, dans le prochain annuaire de l'Ecole des Hautes-Etudes.

Mes travaux et quelques autres petits empêchements me font renoncer à passer quelques jours à Genève maintenant. J'aurais eu, si j'avais pu venir, à vous parler de ma thèse, moins au sujet du travail lui-même, qui vous est déjà connu, qu'en ce qui concerne l'intervention et la décision de la Faculté. Monsieur Ferdinand de Saussure, auquel je l'aurais remise, avait approuvé vivement le sujet choisi et m'avait donné lui-même les principales directions linguistiques qui en ont fait la base. Mes autres directeurs d'études ont été $\mathrm{M}$. Meillet et vous-même, comme vous le savez mieux que personne, et comme je ne l'oublie pas.

La thèse sera prête à être présentée dans une année environ, mais il est possible que ce soit seulement dans deux ans. Je ne puis me rendre aucun compte du temps qu'il me faudra encore. M. Meillet paraît pressé que le travail soit terminé et fasse place à autre chose. Je le suis aussi.

La question de la commission d'examen me laisse très perplexe et assez craintif. Vous devinez pourquoi. D'ailleurs, les professeurs philologues et les discussions théologiques sont un peu à redouter. Ces dernières, pourtant, seraient tout-à-fait hors de leur place dans une Faculté des Lettres, surtout pour une thèse linguistique et non philologique! J'aimerais bien avoir comme examinateurs des linguistes. Il est, surtout, impossible de prévoir l'attitude du professeur de philologie grecque, qui serait charmante pour bien des raisons, mais qui, pour d'autres, pourrait être tout-à-fait désagréable ${ }^{8}$. Je sais que Mr Nicole attend depuis longtemps pour me parler de mes travaux. Une visite qui est venu couper court à la mienne l'a empêché de m'interroger et de me parler suffisamment. J'en étais enchanté, car il eût cherché à me mettre sur une toute autre voie que celle où je voulais marcher sous la conduite de MM de Saussure, Meillet et Bally, mes maîtres préférés! Sachant combien vous m'êtes dévoué, je suis sûr que vous aurez à cœur de défendre mes intérêts le mieux possible. Peut-être, aurez-vous une fois l'occasion de signaler le travail qui vous sera présenté, à un ou deux professeurs qui pourraient être nommés avec vous membres de la commission d'examen. C'est ce que vous saurez mieux que moi. En fin, -quand le moment sera venu, car il ne l'est pas encore,- il pourrait être bon que je vous remette mon travail accompagné d'un certificat de $\mathrm{M}$. Meillet

Vous seriez bien aimable de penser avec moi à ces difficultés et de m'informer, cas échéant, de ce que je dois faire vis-à-vis de la Faculté, bien que je croie les démarches faites tout-à-fait suffisantes pour le moment. Je n'attends pas de réponse à ces lignes, à moins que vous n'ayez, sur ce sujet ou sur un autre, quelque communication pressée à me faire. Je vous sais superlativement occupé.

M. Meillet a aimablement présenté mon nom à la Société de Linguistique et je pense être élu membre de la Société, dans la séance du mois prochain. Je ne trouve pas très glorieux d'y entrer sas avoir fait de publication. Mais cela n'est pas sans précédent. Lorsque M. Meillet, lui-même, y a été admis, en 1889, il n'avait encore rien publié, m’a-t-il dit!

Veuillez recevoir, Monsieur et cher Maître, l'expression de mes sentiments respectueusement dévoués et de ma reconnaissance affectueuse:

Votre dévoué

Paul-F. Regard.

195, Rue de l’Université, Paris VII

BGE : Ms. fr. 3957/06 f. 6-99.

Voilà donc la situation académique de Regard en mars 1916, alors qu'avec Meillet il conçoit l'idée de publier ses notes du deuxième cours de linguistique générale dans les Mémoires de la Société de linguistique, accompagné d'un article ${ }^{10}$.

8 Jules Nicole, célèbre papyrologue genevois.

9 Je remercie la Conservatrice du Département des manuscrits de la Bibliothèque de Genève, Barbara Roth, de m’avoir permis la consultation et publication de cette lettre.

10 Ce n'est qu'en 1958 qu'on aura pu lire l'Introduction de ce deuxième cours dans la reconstruction de Robert Godel qui combine les notes de plusieurs étudiants (CFS 15). Les notes de Regard restent encore méconnues étudiants. 


\section{Le successeur à la chaire de Linguistique à Genève}

Est-ce de sa propre initiative, est-ce sur «l'instigation de Meillet», comme le pense R. Amacker (1989, 102), que Regard fait part de ce projet à Charles Bally? Le fait est que ce dernier ne voit pas de bon oil un projet qui concurrence ses propres vue sur l'héritage saussurien, et sa réaction est immédiate: il rend visite à la veuve de Saussure pour «la mettre au courant». Dans son article «Cent ans de philologie saussurienne», Estanislao Sofia explique quelques détails supplémentaires de «la manière d'agir Bally dans cette affaire» (Sofia, 2013), manière peu digne, certes, mais efficace, comme en témoignent les lettres suivantes.

Marie de Saussure écrit à Meillet le 25 mai une lettre, délicate mais explicite, où elle exprime son oppositionàunequelconque publication «hâtive» des idées de son mari. Signalons que Meillet ne lui avait pas touché mot du projet de Regard. La lettre de Meillet du 6 mars 1913, à laquelle Marie de Saussure «répond», ne mentionne pas du tout le projet de publication, c'est une petite note de condoléances, qui complète celle que Meillet avait écrite auparavant, le 24 février 1913:

\section{5, rue d'Alésia, XIVme}

\section{Chère Madame}

En vous écrivant l'autre jour les quelques lignes auxquelles vous avez bien voulu répondre, je n'ai pu exprimer que mon sentiment personnel.

Je tiens maintenant à vous dire quelle douleur est éprouvée [par] tous les membres de notre petit groupe linguistique de Paris qui était habitué à considérer F. de Saussure comme son maître un peu lointain, mais toujours présent. Nous nous sentons tous diminués par cette perte.

J'essaierai de dire dans le Bulletin de la Société de Linguistique et dans l'Annuaire de
l'Ecole de Hautes Etudes qui paraîtront vers octobre ou novembre ce que F. de Saussure a été pour nous.

En attendant, je vous prie de croire que nous sommes ici tout un petit groupe qui partage votre douleur et d'agréer mon respectueux hommage.

\section{A. Meillet}

Paris, 6 mars

Lettre inédite, manuscrit appartenant à Jacques et Philippe de Saussure et déposée au Château de Vufflens (Mejía Quijano, 2015).

Et voici maintenant la lettre de Marie de Saussure à Antoine Meillet, connue depuis sa publication par Emile Benveniste en 1964, mais dont on ne connaissait pas le contexte:

\section{Cher Monsieur}

Vous m'excuserez cette fois encore de venir si tard vous remercier de votre aimable lettre du 6 mars, ainsi que du témoignage si affectueux que vous avez rendu à la mémoire de mon mari à l'ouverture de vos cours au Collège de France et à l'Ecole des Hautes-Etudes. -Je ne puis vous dire combien j'y suis sensible et c'est de tout mon cœur aussi que je m'associe à tous les regrets qui m'ont été exprimés au nom de la science à l'occasion de la mort de mon mari ${ }^{11}$.

Je sais avec quel intérêt il suivait les études et les travaux de ceux qui lui succèdent dans la carrière et vous savez que rien dans ce domaine ne lui était indifférent. -Il a donné le meilleur de son temps à son enseignement, et la préparation de ses cours l'absorbait au point de l'empêcher de publier d'autres travaux personnels-.

Et maintenant plusieurs de ses élèves $m$ 'ont demandé s'il n'y aurait pas, dans ses notes, quelque chose à publier. Ce serait naturellement chose à voir et à laquelle je ne voudrais pas

$11 \mathrm{Au}$ Château de Vufflens se trouvent des centaines de missives que Marie de Saussure et ses fils ont reçues à l'occasion de la mort de Ferdinand de Saussure, auxquelles on peut ajouter un bon nombre d'autres lettres semblables conservées à la BGE. Dans mes recherches documentaires, je n'ai jamais vu une telle quantité de lettres de condoléances, dont quelques-unes obéissent naturellement au simple devoir social, mais dont la plupart sont sincèrement écrites et extrêmement émouvantes. 
m'opposer d'emblée; mais, cher monsieur, vous avez assez connu mon mari, pour savoir avec quelle scrupuleuse conscience il abordait chaque question et j'ai l'impression qu'il faudrait en tous cas ne rien publier trop vite car je ne voudrais rien faire qu'il n'eût pas approuvé.

Peut-être qu'en compulsant les notes prises par différents élèves en différentes années pourrait-on avoir une idée assez complète d'un de ses cours, mais pour cela aussi il ne faut rien presser. -N'est-ce pas aussi votre avis-. On peut quelquefois par une publication trop hâtive, déflorer un travail qu' on pourrait avec le temps faire plus complet. Je suis, naturellement, incompétente dans cette question, cependant je sais que mon mari n'agissait jamais avec précipitation et que ce qu'il a donné à la science était le fruit de travaux mûrement réfléchis. - J'avais l'intention de réunir dans une petite brochure les articles et témoignages reçus à l'occasion de la mort de mon mari, mais je veux attendre pour cela que les articles à venir aient paru.

J'espère pouvoir me procurer plus tard ceux que vous ferez paraître dans les Mémoires de la société de linguistique et l'Annuaire de l'Ecole des Hautes Etudes. J'ai pensé que ce serait un souvenir pour la famille et les amis- ${ }^{12}$.

J'espère, Cher Monsieur, que si vous veniez une fois de nos côtés, vous me ferez le plaisir de venir me voir. -Je serais toujours heureuse de serrer la main à ceux qui se sont associés de cour à notre grand deuil.

Recevez, Cher Monsieur, mes meilleurs compliments

Marie de Saussure

Tertasse 2, Genève

le 25 Mai [1913]

Lettre publiée par Emile Benveniste à la fin de sa correspondance Meillet-Saussure et reproduite ici telle quelle. (Benveniste, 1964).
Nous ne connaissons pas la réponse de Meillet à cette lettre de Marie de Saussure, mais entretemps Bally avait écrit à Regard en le mettant au courant de l'opposition de la veuve de Saussure, comme l'affirme Regard dans cette lettre à Marie de Saussure, à ce propos:

Paris VII, 195 Rue de l'Université

Le 27 mai 1913

Très honorée Madame,

Il vous a été parlé, je ne sais en quels termes mais -certaines choses le font craindre- d'une façon assez inexacte et, en tout cas, prématurée, du projet de Monsieur A. Meillet et de l'article (fait sous sa direction et celle de M. Bally) qu'il désirait faire paraître dans les «Mémoires de la Société de Linguistique de Paris» (Volume XIX) pour des motifs importants, article qui eût été fait (il faut le dire) avec les soins les plus scrupuleux et toutes les précautions nécessaires.

M. Bally m'a écrit, il y a quelques jours, une lettre dans laquelle il me dit que vous écrivez à Monsieur A. Meillet et me laisse entendre (il est vrai sous une forme un peu vague) que vous vous opposez à son projet. M. Meillet me dit, ce matin, qu'il n'a pas reçu de lettre de vous.

Je crois devoir vous dire (en mon propre nom, car ce n'est pas un message dont j'aie été chargé) qu'il renonce tout-à-fait à ce projet, étant donné le tour qu'ont pris les choses à Genève. (Je l'avais informé par lettre télégraphique que, pour ma très modeste part, je l'abandonnais complètement, sous la seule réserve de votre désir et de son opinion).

Je ne puis ni vous en dire plus long maintenant sur la pensée de Monsieur A. Meillet, ni prendre, comme élève de Monsieur F. de Saussure, une responsabilité quelconque sur les conséquences que risque d'avoir la méconnaissance de l'avis de Monsieur A Meillet, dont personne n'a la compétence à l'heure actuelle. Si vous avez quelque question à me poser, je me mets entièrement à votre disposition pour y répondre dans toute la mesure possible.

12 Cette brochure, très intéressante, a été connue par la suite sous la dénomination «Plaquette d'hommage» et a été rééditée par les fils de Saussure en 1963. Quelques exemplaires de ce petit livre, aujourd'hui introuvable, se trouvent au Château de Vufflens (Mejía Quijano, 2015). 
Je me propose, d'ailleurs, de passer deux mois en Suisse cet été, et si c'est un entretien oral que vous désirez, je me rendrai volontiers auprès de vous.

Le projet assez vaste qui a été fait par $M$. Bally et quelques autres personnes (et auquel j'avais pensé moi-même) est très séduisant. Mais la réalisation d'une telle entreprise sera certainement extrêmement difficile, car elle offre bien des dangers inquiétants. Mon désir personnel est, maintenant, qu'il réussisse dans la mesure où vous l'avez fait vôtre et où il pourra être couronné de succès.

Monsieur F. de Saussure était mon maître le plus aimé, le plus admiré, le seul auquel une affection et une confiance illimitée allaient sans réserve aucune. Sa perte (que je ressens tout particulièrement) m'est une douleur très cruelle et me laisse inconsolable...

Veuillez, très honorée Madame, excuser la liberté de ces lignes dictées par le devoir et croire à mes sentiments très profondément respectueux et très sincèrement dévoués:

\section{Paul-F. Regard.}

Lettre inédite, manuscrit appartenant à Jacques et Philippe de Saussure et déposé au Château de Vufflens (Mejía Quijano, 2015).

Voilà donc que le projet de publier les notes d'un étudiant ayant assisté aux cours de linguistique générale de Saussure a fait long feu face à un autre projet, où c'est un tiers extérieur aux cours qui est censé rédiger la pensée du maître. En essayant de pousser tous les pions, Bally avait écrit aussi à Antoine Meillet une lettre où il met en doute, sans aucune preuve directe, comme il l'avoue luimême, la qualité des cahiers de Regard:
Genève 29 mai 1913

Mon cher confrère,

Vous sachant en voyage, je n'ai pu vous entretenir directement d'une question que j'aurais voulu traiter avec vous seul. Pensant que vous êtes de retour je m'empresse de vous informer de ce qui s'est passé, afin que ma manière d'agir ne crée aucun malentendu. Je n'ai pas suivi moi-même l'enseignement de Saussure sur la linguistique générale et ne le connais que par des notes- admirablement établies -d'un de ses élèves qui en a profité pendant deux années ${ }^{13}$. Dès que j'ai eu connaissance de l'intéressant projet communiqué par $\mathrm{M}$. Regard, je me suis renseigné auprès des autres élèves de $S$., en particulier Léop. Gautier et A. Séchehaye. Sans rien leur dire du projet d'article, pour ne pas les influencer, j'ai recueilli leur avis sur la nature du cours et les conditions les plus favorables d'une publication éventuelle. Leurs vues concordent exactement sur tous les points suivants: sans que les principes de l'enseignement aient varié ${ }^{14}$, chaque cours annuel (au nombre de trois) a son caractère propre et sa note originale, et beaucoup de détails de chacun d'eux peut enrichir les deux autres sans leur faire tort. Toutes les personnes consultées sont convaincues que l'œuvre vaut par l'ensemble et la systémisation autant que par les parties séparées; toutes insistent sur ce point: quel que soit le mode de publication à adopter, le travail ne peut être fondé sur les notes d'un seul élève auditeur d'un seul des trois cours; on pense que, avant de songer à un article, on devrait envisager la possibilité d'un ouvrage séparé, quitte à abandonner cette idée si elle ne paraitt pas réalisable.

Madame de Saussure à qui j'ai rendu visite la semaine dernière pour la mettre au courant, m'a appris que MM. Séchehaye et Leop. Gautier lui avaient parlé déjà de leurs idées sur ce sujet ${ }^{15}$, et qu'elle est disposée à ne pas prendre de décision avant l'enquête mentionnée ci-dessus. Enfin je dois ajouter un renseignement confidentiel, très important, et qui restera, si vous le voulez bien,

13 Comme le remarque Estanislao Sofia, il ne saurait s'agir que d'Albert Riedlinger, dont les notes, maintenant que l'on connaît des notes d'autres étudiants et qu'elles peuvent donc être autrement appréciées, sont loin d'être aussi «admirablement établies» que le proclame Bally, lequel, rappelons-le, n'avait assisté à aucun cours de linguistique générale (Sofia, 2013).

14 Un siècle après, et grâce à la publication de notes des étudiants des trois cours, on peut aisément contester cette affirmation.

15 Un troisième projet dont on ne connaît pas la teneur? 
entre nous: j'ai appris de bonne source, par une personne qui a lu les notes de M. Regard, que ces notes, toutes consciencieuses qu'elles sont, ne rendent pas l'esprit de l'enseignement de S., et le défigurent même complètement par endroits. Je ne puis contrôler cette information, mais elle concorde avec mon impression sur les méthodes de travail de M. Regard, propres à saisir les détails plutôt que l'ensemble d'une question.

Tout cela me donne sérieusement à réfléchir; j'espère, mon cher confrère, que vous ne verrez dans ma manière d'agir que le désir de sauvegarder une mémoire que nous respectons tous; peut-être vaut-il mieux différer un peu l'exécution pour que celle-ci ne ménage pas de surprises désagréables. Si vous m'y autorisez, je vous tiendrai au courant de ce que j'entreprendrai sur tout cela. Dans ce moment, je m'occupe activement à recueillir les notes d'élèves, et j'espère toujours que vous ne nous refuserez pas vos précieux conseils.

Etes-vous content de votre voyage? Gauthiot est-il déjà parti?

Amitiés à tous, s'il vous plaît.

Dans quelque temps je pense pouvoir vous envoyer mon petit livre «Le langage et la vie». Recevez, mon cher confrère, mes bien cordiales salutations.

Ch. Bally

P. S. Excusez ce griffonnage; je vous écris très à la hâte.

Lettre publiée par René Amacker, en collaboration avec Simon Bouquet (1989), avec quelques notes explicatives ${ }^{16}$.

Meillet, répond à Bally en signalant leur décision de renoncer au projet contesté, mais ne manque pas aussi de préciser son point de vue théorique sur la question:

Mon cher confrère

Je suis en effet de retour depuis lundi dernier. Gauthiot est parti pour son voyage, et je trouve Paris vide sans lui. Comme je l'ai écrit à Madame de Saussure, le projet que j'avais esquissé avec le jeune Regard est abandonné; ce projet a toujours été subordonné à votre agrément, et, dès l'instant que vous avez d'autres vues, il ne doit pas en être question.

Il m'est malaisé d'apprécier le projet dont vous me parlez. En principe, j'ai de très gros scrupules contre les publications posthumes, et c'est en grande partie pour cela que j'avais indiqué à Regard le projet que vous savez. J'aurais peut-être un peu plus de scrupules encore contre la contamination de cours divers. Mais vous êtes mieux placé que moi pour apprécier les choses, et vous disposez d'éléments d'appréciation qui me manquent. En tout cas, je vous serai toujours très obligé de me faire savoir ce que vous avez décidé, quand vous aurez arrêté un projet définitif.

J'ai été content de mon voyage, qui m'a reposé et diverti autant que je pouvais l'espérer.

Votre bien cordialement dévoué

A. Meillet

31 mai

Lettre publiée par René Amacker, en collaboration avec Simon Bouquet (1989).

\section{L'enjeu théorique}

C'est ce point de vue de la «contamination», mentionnée par Meillet, qu'il me semble intéressant de remarquer maintenant, un siècle après la parution du projet de Bally, car on peut constater que d'autres linguistes en sont venus à réaliser, pour leur part et tout à fait spontanément, le projet de Regard-Meillet, à savoir la publication des notes des étudiants de Saussure.

Rétrospectivement, l'enjeu théorique de ce projet s'avère donc dépasser les contingences historiques des personnes impliquées, car il concerne le corps théorique de la linguistique saussurienne. Rappelons que les principes généraux proposés par Saussure dans son troisième cours portent

16 Je tiens à préciser que, dans un article précédent (2016), j'ai par erreur attribué ces commentaires explicatifs au seul Simon Bouquet. 
notamment sur l'arbitraire du signe, la distinction langue-parole et la dualité synchronie-diachronie. L'arbitraire du signe est le garant de la multiplicité de sens possibles pour les combinaisons linguistiques. Une combinaison donnée n'a de sens délimité que par les variables de l'acte de parole, dont l'identité des interlocuteurs et les coordonnées spatio-temporelles délimitent en particulier une synchronie précise. Or, sur la base de ces principes, on peut comprendre un texte comme le produit d'un acte de parole précis appartenant à une synchronie. La contamination des trois cours ne pouvait, par conséquent, que brouiller la pensée exposée par un tiers étranger aux actes de parole originaires.

C'est du fait que, digne disciple de Saussure, Regard comprend parfaitement la distinction synchronie-diachronie et ce que signifie la synchronie concernant un texte, comme il l'aura démontré dans sa thèse de 1918, que, même ayant renoncé à son propre projet, il écrit de nouveau à Marie de Saussure à ce propos, en essayant de limiter les dégâts qu'il entrevoit dans le projet de Bally, à savoir, la défiguration de la voix et de la pensée de son maître bien-aimé:

Paris, le 9 juin 1913

Très honorée Madame,

Permettez-moi, je vous prie, de vous adresser mes remerciements les plus empressés pour vos lignes si aimables auxquelles j'ai été très sensible.

Le projet dont vous voulez bien me parler obtiendra sans doute une approbation unanime si M. Bally consent à mettre de côté son idée de contamination de plusieurs cours telle qu'il l'a présentée dans ses lettres à Paris. C'est dans l'œuvre même de Monsieur Ferdinand de Saussure que ses élèves ont à trouver le moyen qui convient à l'exposition fidèle des idées si riches et si fécondes de leur maître bien aimé. La communication sur l'Accentuation lituanienne dans les Indogermanische Forschungen VI Anzeiger p. 157 sqq. me parait être, par excellence, le modèle à suivre, en principe, dans le travail qu'ils se proposent.

Le projet de publication peut se concevoir de façons assez diverses. Et, suivant les limites qu'on jugera convenable de lui assigner, il y aura beaucoup de choses dont on pourra tirer le meilleur parti et d'autres qu'il faudra laisser avec le plus grand regret. Ce que je vois maintenant, pour ma très modeste part, c'est un livre orné d'un portrait (celui, je pense, qui a paru en 1908 dans la Patrie Suisse avec un ravissant article de M. Bally et qui a été reproduit assez récemment dans la Semaine littéraire avec un long et splendide article du même auteur) et comprenant, avec une préface, un nombre à fixer d'articles qui pourront être empruntés à autant de cours que l'on voudra mais sans jamais les mélanger entre eux (amendement au projet actuel de M. Bally).

Ceux qui travailleront au livre devront, me semble-il, avoir la préoccupation constante de montrer comment Monsieur Ferdinand de Saussure était à la fois un linguiste génial et un très remarquable poète. Nul, en effet, n'a eu à un plus haut degré ce que Pascal appelait l'esprit de géométrie et l'esprit de finesse.

Si l'on agit ainsi, les idées de Monsieur Ferdinand de Saussure seront propagées en conservant la marque personnelle de leur auteur.

Il me paraît prématuré de parler d'autres questions maintenant. Mais un point très important (car il pouvait modifier même le projet général) est de savoir tout de suite dans quelle mesure Monsieur Ferdinand de Saussure a laissé des manuscrits dont vous voudriez bien permettre l'utilisation en vue du livre consacré à sa mémoire.

Comme vous m'y invitez dans vos très aimables lignes, je serai enchanté, Madame, de vous rendre visite pendant mon séjour en Suisse.

Je vous suis superlativement reconnaissant de la bienveillante pensée qui m'en procure l'occasion.

Veuillez agréer, très-honorée Madame, l'expression de ma gratitude bien vive et de mes hommages les plus respectueusement dévoués:

$$
\text { Paul-F. Regard. }
$$

Lettre inédite, manuscrit appartenant à Jacques et Philippe de Saussure et déposé au Château de Vufflens (Mejía Quijano, 2015). 
Cette lettre de Regard témoigne de l'importance de cet épisode: il s'agit de respecter la base méthodologique de la dualité synchronie-diachronie introduite par Saussure, car les trois cours constituent une diachronie, et les mélanger revient en fait à effacer la pensée même du professeur, que cette diachronie contient, une pensée toujours en mouvement, dynamique et inventive, comme les langues humaines. Comme l'affirme Regard, à la fin de l'introduction de sa thèse, Saussure a surtout donné à ses étudiants une méthode, sa théorie est une somme de principes méthodologiques qu'il est bon de suivre si l'on veut aborder avec fruit n'importe quel problème linguistique.

\section{La force des choses}

Après la publication du CLG, Meillet et Regard ne manqueront pas de s'exprimer, chacun de son côté, concernant l'intromission d'un tiers, entièrement extérieur à la pensée transmise dans les cours de linguistique générale de Saussure, en insistant sur la déformation inhérente à la contamination des cours que le projet de Bally comportait dès le début:

[Les éditeurs] ont pris le parti hardi de fondre en un tout les trois rédactions et de construire, pour ainsi dire, avec les formules et les exemples de F. de Saussure, le livre que le maître n'avait pas fait, qu'il n'aurait sans doute jamais fait. Ce qui est offert au public, c'est donc une rédaction des idées de F. de Saussure sur la linguistique générale par ses deux principaux élèves genevois, MM. Bally et Sechehaye. [...] Il n'y a pas lieu d'entrer ici dans la critique de détail d'un livre qui n'est que l'adaptation d'un enseignement oral fugitif, et où l'on ne sait si les détails qui seraient critiquables viennent de l'auteur ou des éditeurs. (Meillet, 1916: 32-33).

$\mathrm{Au}$ prix de quelques redites, la publication des notes de cours n'aurait-elle pas conservé plus fidèlement la pensée de F. de $S$., avec sa puissance, avec son originalité? Et les variations elles-mêmes que les éditeurs paraissent avoir craint de mettre au jour n'auraient-elles pas offert un intérêt singulier? (Regard, 1918: 11-12).

On pourrait affirmer ironiquement que la décision d'une veuve sans connaissances linguistiques a modelé la linguistique du XXème siècle, mais il est vain de regretter le passé; il vaut mieux, à partir de la connaissance lucide du passé, de construire au présent le futur.

Constatons ainsi que depuis les travaux de Robert Godel (1954, 1957, 1958) et l'édition critique de Rudolf Engler du Cours de linguistique générale (1968-1974), c'est en fait la réalisation du projet de Regard-Meillet qui a occupé les plus grands efforts des linguistes saussuriens, et cela de façon spontanée aux plusieurs points de la planète: au Japon (entre autres, avec l'équipe de feu Eusuke Komatsu), en Suisse (avec les études du regretté Johannes Fehr et mes propres publications qui suivent un projet de 1990 conjoint avec Rudolf Engler), en Colombie (avec l'équipe du groupe SEMSA). Ces travaux d'édition des notes des étudiants en français ont été relayés par des traducteurs en anglais (Roy Harris, George Wolff), en allemand (Annette Kaudé), en japonais (Katzuhiro Matsuzawa), en coréen (Sung Do Kim), et prochainement en espagnol colombien.

Un tel accord spontané sur la nécessité de réaliser ce projet concurrent du CLG, est-il gratuit ou, peut-être bien, tout comme fut le cas pour Meillet et Regard, tient-il à l'assimilation d'une partie de l'enseignement saussurien, permise au XXème siècle par l'étude du même CLG?

\section{Références}

Amacker, René, en collaboration avec Bouquet Simon, 1989. «Correspondance Bally-Meillet (1906-1932)» Cahiers Ferdinand de Saussure. 43: 95-127.

Bally, Charles et Sechehaye, Albert 1916. Cours de linguistique générale de Ferdinand de Saussure. París: Payot.

Benveniste, Emile, 1964. «Lettres de Ferdinand de Saussure à Antoine Meillet». Cahiers Ferdinand de Saussure 21: 89-130.

Engler, Rudolf, 1968-1974. Cours de linguistique générale. Edition critique (CLG-E). Wiesbaden: Otto Harrassowitz. 
Fehr, Johannes, 1997. Ferdinand de Saussure: Linguistik und Semiologie. Notizen aus dem Nachlass, Texte, Briefe und Dokumente; Gesammelt, übersetzt und eingeleitet von Johannes Fehr. Frankfurt am Main, Alemania: Suhrkamp.

- 2002. Saussure entre linguistique et sémiologie, traduit de l'allemand par Pierre Caussat. París, Francia: Presses Universitaires de France.

Meillet, Antoine, 1916. "Compte-rendu du Cours de Linguistique Générale", Bulletin de la Société de Linguistique de Paris, Tome XX, 1916, 32-33, 36.

Mejía Quijano, Claudia, 1998. La linguistique diachronique: le projet saussurien. Publications du Cercle Ferdinand de Saussure. Ginebra, Suiza: Librairie Droz.

- 2006. "Sous le signe du doute". Cahiers Ferdinand de Saussure, 58: 43-67.

- 2008. Le cours d'une vie. Portrait diachronique de Ferdinand de Saussure. Tome 1: Ton fils affectionné. Nantes, Francia: Editions Cécile Défaut.

- 2012a. "La parole, acte créateur de langue». Bulletin de la Société de linguistique de Paris 107/1: 43-102.

- 2012b. Le cours d'une vie. Portrait diachronique de Ferdinand de Saussure. Tome 2: Devenir père. Nantes, Francia: Editions Cécile Défaut.

- 2014. Ferdinand de Saussure. Une vie en lettres 1866- 1913. Diachronie dressée par Claudia Mejía Quijano. Collection psychanalytique Psyché, dirigée par François Ansermet. Nantes, Francia: Editions nouvelles Cécile Défaut.

- 2015. “L'œuvre en réseaux: nouvelle découverte de manuscrits saussuriens". Beiträge zur Geschichte der Sprachwissenschaft. 25-1: 149176.
Mejía Quijano, Claudia, Jaramillo Giraldo, Daniel y Pérez Zapata, Alexander, 2016. “La part de l'élève dans la parole du maître: Hommage aux élèves de Ferdinand de Saussure à l'occasion du centenaire du Cours de Linguistique Générale". Beiträge zur Geschichte der Sprachwissenschaft. 26-1: 287-306.

Regard, Paul-F., 1918. Contribution à l'étude des prépositions dans la langue du Nouveau Testament, Paris: Leroux.

- 1919. La phrase nominale dans la langue du Nouveau Testament. Paris: Leroux.

Saussure, Ferdinand de,

- 1957. "Introduction au deuxième cours de linguistique générale de Ferdinand de Saussure". Editée par Robert Godel, Cahiers Ferdinand de Saussure 15: 3-103.

- 1968. Cours de linguistique générale. Edition critique par R. Engler.Tomo 1. Wiesbaden, Alemania: O. Harrassowitz.

- 1974. Cours de linguistique générale. Edition critique par R. Engler.Tomo 2, notes autographes. Wiesbaden, Alemania: O. Harrassowitz.

- 1987. Cours de linguistique générale (III) de F. de Saussure. Edité par Eusuke Komatsu. Tokyo, Japón: Faculty of letters, Gakushuin University.

- 1993a. F. de Saussure. Cours de linguistique générale: premier et troisième cours d'après les notes de Riedlinger et Constantin. Edité par Eusuke Komatsu.Tokyo, Japón: Université Gakushuin.

- 1993b. Third Course in General Linguistics. Edité par Eusuke Komatsu et Roy Harris. Oxford, Inglaterra: Pergamon.

- 1996. F. de Saussure. Premier cours de linguistique générale (1907), d'après les cahiers d'Albert Riedlinger. Edité par Eusuke Komatsu et Georges Wolf. Oxford, Inglaterra: Pergamon. 
- 1997. F. de Saussure. Deuxième cours de linguistique générale: (1908-1909), d'après les cahiers d'Albert Riedlinger et Charles Patois. Edité par Eusuke Komatsu et George Wolf. Oxford, Inglaterra: Pergamon.

- 2006a. “Emile Constantin. Cours delinguistique générale de $\mathrm{Mr}$ le professeur Ferdinand de Saussure 1910-1911". Edité par Claudia Mejía Quijano avec la collaboration de Claude Sandoz. Cahiers Ferdinand de Saussure 58: 83-289.

- 2006b. “Notes préparatoires pour le cours de Linguistique générale 1910-1911". Editées par Daniele Gambarara Cahiers Ferdinand de Saussure 58: 92-259.

- 2006c. Kaudé, Annette. Saussure letztes Wort. Deutsche Übertsetzung und Deutung der Hörermanuskripte zur dritten Genfer Vorlesung über allgemeine Sprachwissenschaft (1910-1911). Tesis de la Universidad de Dusseldorf. Consultado en http:// docserv.uni-dusseldorf.de/servlets/ DerivateServlet/Derivate- 3481/1481.pdf.

- 2017a. Troisième cours de linguistique générale, Ecrits et leçons de "Linguistique générale" de Ferdinand de Saussure IV, traduit en japonais et annoté par Kazuhiro Matsuzawa, Tokyo, Editions Iwanamishoten (à paraître).

- 2017b. Le Troisième cours de linguistique générale, traduit en coréen et annoté par Sung Do Kim (à paraître).

- 2017c. Lingüística general. El Primer Curso. Intégralement edité et traduit en espagnol colombien par Claudia Mejía Quijano, Daniel Jaramillo Giraldo y Alexander Pérez Zapata, (à paraître).

Saussure, Marie de (ed.), Ferdinand de Saussure, Plaquette d'Hommage. 1913, hors commerce.

Sofia, Estanislao 2013. “Cents ans de philologie saussurienne". Cahiers Ferdinand de Saussure 66: 181-197.

Je remercie Messieurs Philippe et Jacques de Saussure pour m'avoir permis de publier les images et les lettres envoyées à Marie de Saussure.

Les manuscrits du Château de Vufflens ont été découverts lors d'une recherche financée par l'Ecole des langues de l'Université d'Antioquia (UdeA; calle 70 № 52-21, Medellín-Colombia). 